\title{
Statut et usages de la notion de genre en didactique(s) : retour sur quelques propositions
}

Yves Reuter

\section{(2) OpenEdition}

Journals

Édition électronique

URL : http://journals.openedition.org/pratiques/3818

DOI : $10.4000 /$ pratiques.3818

ISSN : 2425-2042

Éditeur

Centre de recherche sur les médiations (CREM)

Édition imprimée

Date de publication : 1 juin 2013

Pagination : 153-164

Référence électronique

Yves Reuter, «Statut et usages de la notion de genre en didactique(s) : retour sur quelques propositions », Pratiques [En ligne], 157-158 | 2013, mis en ligne le 18 décembre 2017, consulté le 02 mai 2019. URL : http://journals.openedition.org/pratiques/3818; DOI : 10.4000/pratiques.3818

(c) Tous droits réservés 


\section{Statut et usages de la notion de genre en didactique(s) : retour sur quelques propositions}

\section{Yves Reuter}

Université de Lille 3 - Theodile-CIREL (E.A. 4354)

Cette contribution ${ }^{(1)}$ s'organise autour de trois entrées : les raisons qui avaient amené notre équipe de recherche à constituer la notion de genre scolaire / didactique, associée à celle de pratiques, comme objet de recherche, pendant quatre ans (de 2003 à 2007), au travers de son séminaire mensuel; les principes essentiels qui, dans le cadre théorique didactique qui est le nôtre, ont régi sa construction; les usages que nous en avons fait et que nous en faisons, appuyés sur quelques exemples. Je conclurai cette présentation, sans nul doute trop succincte, par quelques problèmes méthodologiques auxquels nous nous confrontons encore.

\section{Les conditions d'une émergence}

Trois raisons principales contribuent, à mon sens, à expliquer l'existence d'un programme consacré au genre : certains éléments du contexte théorique, la récurrence de cette notion lors de nos recherches antérieures, ses intérêts pour le projet de connaissance des didactiques.

(1) Cet article constitue une version sensiblement remaniée et actualisée de mon article du Français aujourd'hui, intitulé " Statut et usages de la notion de genre en didactique(s) : quelques propositions » $\left(\mathrm{n}^{\circ} 159,2007\right.$, p.11-18). Je remercie les éditions Armand Colin, le directeur de la revue Jacques David ainsi que les coordinatrices du numéro, Catherine Boré et Isabelle Laborde-Milaa, de m'avoir autorisé à reprendre ce texte. Je précise encore que cette première version était elle-même une version transformée de ma communication à la journée d'étude CEDITEC-Theodile, Le genre comme outil, comme objet, comme enjeu, qui s'est tenue à l'université de Paris 12, le 9 décembre 2005, sous la direction d'Isabelle Laborde-Milaa. De plus cette contribution a bénéficié des échanges constants avec les membres de l'équipe Theodile pendant la durée du programme mentionné. Qu'ils en soient ici remerciés. Ces conditions d'élaboration expliquent, au moins en partie, les oscillations entre le « je » et le « nous» dans cet article. 


\subsection{Quelques éléments du contexte théorique}

Si le genre n'avait jamais véritablement disparu du paysage théorique en sciences humaines, on avait pu néanmoins constater un retour en force de cette notion, notamment dans le domaine des études linguistiques et textuelles (par exemple, Adam 1999), domaine dont la dimension contributoire est importante, pour la didactique du français en particulier et pour les didactiques en général, si l'on accepte l'idée que l'étude du fonctionnement des pratiques langagières au sein des différents enseignements disciplinaires représente une composante essentielle de leurs investigations (Delcambre 2007/2010 a et b).

De manière plus spécifique, au sein même du champ des recherches en didactiques, on peut remarquer que certaines équipes ont fait du genre une pièce-maîtresse de leur édifice théorique. C'est le cas, me semble-t-il, des didacticiens du français de l'université de Genève qui articulent cette notion, construite en référence à la dichotomie « bakhtinienne » ${ }^{(2)}$ de « genres premiers/genres seconds », aux thèses sur l'apprentissage de Vygotski, constituant ainsi le genre en outil culturel, en outil d'enseignement et d'apprentissages, en objectif et en objet d'enseignement et d'apprentissages ${ }^{(3)}$. C'est encore le cas, pour prendre un autre exemple, des didacticiens qui travaillent à Bordeaux autour de Jean-Paul Bernié pour qui l'« entrée » dans une discipline, considérée comme un ensemble de manières spécifiques de penser, de parler et d'agir, est indissociablement, une entrée dans un univers culturel caractérisé par des genres singuliers (voir, par exemple, Bernié 2000 ; Jaubert, Rebière et Bernié 2003, ou encore Jaubert 2007).

\subsection{Les programmes de recherche antérieurs de Theodile}

Ces dimensions contextuelles n'auraient sans doute pas été suffisantes si nos programmes de recherche antérieurs ainsi que l'ensemble des recherches que nous avions pu mener ne nous avaient pas confrontés systématiquement aux catégories de genres (et de pratiques). En effet, dans les trois programmes pluri-annuels précédents, consacrés respectivement aux interactions entre lecture(s) et écriture(s) ( $c f$. Reuter éd. 1994 ; Pratiques 1995 ...), à la description dans les différentes disciplines scolaires ( $c f$. Reuter éd. 1998 ; Pratiques 1998...) et au rapport à l'écriture et à l'image du scripteur (cf. Enjeux 2001 ; Pratiques 2002...), nous avions été amenés à nous servir, de manière récurrente, de la notion de genre, envisagée notamment comme un instrument permettant de penser :

- l'articulation entre les pratiques : entre les pratiques de lecture et d'écriture, par exemple ;

- la spécification des produits et des productions : la contextualisation et l'actualisation singulière des types d'écrits ou d'activités, les positions du scripteur attendues, les modalités descriptives sollicitées...;

— la différenciation des objets et/ou des pratiques : par exemple, les genres lus mais non écrits par les élèves ou l'inverse, ou encore les variations dans

(2) Les guillemets renvoient à la mise en cause, particulièrement étayée, de l'attribution de nombre de travaux à Bakhtine (Bronckart et Bota, 2011).

(3) Voir, entre autres, Schneuwly (1994) pour une présentation générale de ce cadre et Dolz et Schneuwly (1998) pour une mise en œuvre plus spécifique sur l'oral. 
les descriptions ou les images du scripteur selon les cadres génériques disciplinaires.

\subsection{Le projet de connaissance des didactiques}

Nous avons été attentifs à ces éléments dans la mesure où la notion de genre nous a paru pouvoir constituer un outil théorique particulièrement pertinent au sein du projet de connaissance des didactiques : décrire, comprendre et analyser les fonctionnements disciplinaires, les contenus (savoirs, savoir-faire...) en tant qu'ils sont des objets d'enseignement et d'apprentissages référés/ référables à des disciplines (Reuter, dir. 2007/2010). Nous y avons été aussi attentifs dans la mesure où notre laboratoire a toujours accordé une importance particulière à la question des pratiques langagières au sein des disciplines et à celle des contenus comme productions et produits langagiers. Nous y avons été sans doute d'autant plus sensibles que nous sommes particulièrement attachés, au sein de Théodile, à la spécification des didactiques en tant que disciplines de recherche, ce qui implique une exigence «épistémologique », une réflexion constante quant au corps conceptuel (Reuter, dir. 2007/2010) tout autant qu'aux méthodes mises en œuvre (Perrin-Glorian, Reuter, dir., 2006 ; Lahanier-Reuter, Roditi, dir. 2007 ; CohenAzria, Sayak, dir. 2009).

\section{Principes de construction de la notion de genre}

Je me contenterai, ici encore de façon bien trop cavalière, de préciser les principes de constitution de la notion de genre qui me sont apparus essentiels dans une perspective didactique.

\subsection{L'actualisation du genre selon les « espaces"}

Le genre me parait à analyser, fondamentalement, en tant qu'élément constitutif des fonctionnements au sein des espaces ${ }^{(4)}$ des pratiques didactiques (pour nous, essentiellement au sein de la classe ou de ce qui en dépend ${ }^{(5)}$ ), de la prescription (par exemple, en France, dans les Instructions Officielles) et de l'encadrement des pratiques (sollicitations et recommandations des formateurs, des inspecteurs, des manuels, des associations de spécialistes ou de militants...) et des représentations des acteurs.

Ce principe correspond à un mode d'éclairage singulier : considérer les disciplines comme des constructions au mode d'existence variable selon les espaces d'actualisation. Cela implique comme conséquence le fait de considérer que la notion de genre renvoie à des référents hétérogènes. Ou, sous une autre forme, que les genres ont des modes d'existence différents. En effet, ainsi que nous 1'avions effectué à propos des « images du scripteur» (Delcambre et Reuter 2002)

(4) J'ai formalisé progressivement la notion d'espaces différenciés et articulés d'actualisation des disciplines, notamment dans ma communication de 2005. Elle est depuis devenue essentielle dans mon approche du fonctionnement des disciplines scolaires.

(5) Le travail à la maison, par exemple. 
ou de la discipline scolaire (Reuter 2004a ; Reuter, Lahanier-Reuter 2004/2007), il me parait indispensable de distinguer, entre autres, le genre en tant que modèle (« savant», institutionnel...), le genre en tant que représentation(s) (dans l'esprit des acteurs), le genre en tant qu'exemple actualisé...

\subsection{Le genre à l'articulation entre les dimensions institutionnelle, pédagogique et disciplinaire}

En tant qu'il est un élément structurel des fonctionnements didactiques, le genre se constitue dans l'interaction entre trois systèmes, étroitement imbriqués ${ }^{(6)}$ : le système scolaire (ce que d'aucuns appellent la forme scolaire ${ }^{(7)}$ ), le système pédagogique (qui organise les formes de l'enseignement et des apprentissages ainsi que leurs relations) et le système disciplinaire (qui organise les contenus à transmettre et certaines modalités de travail les concernant), chacun de ces systèmes pouvant s'actualiser dans des configurations différentes.

Cette perspective permet, entre autres, de distinguer à côté de genres à dominante disciplinaire (la dictée orthographique, le complètement de carte en géographie...), des genres à dominante scolaire (échanges sur les élèves en salle des professeurs, interventions en conseil de classe, bulletins scolaires...), des genres à dominante pédagogique (leçons, exercices, « quoi de neuf ? »...). Elle permet donc de réfléchir à la part de chacune de ces dimensions dans le fonctionnement des genres. Elle permet aussi d'éclairer d'éventuelles tensions tributaires des interactions entre ces systèmes (par exemple entre le pédagogique et le disciplinaire lors d'une écriture en projet, longue et collective, qui porterait l'accent sur les fonctionnements de groupe plutôt que sur les dimensions scripturales). Elle permet encore de s'interroger sur le moment du cursus scolaire où genres (et pratiques) deviennent « réellement » disciplinaires.

\subsection{Le genre entre « scolaire » et « extrascolaire»}

Cette constitution des genres au sein des systèmes scolaire, pédagogique et disciplinaire, n'implique cependant pas une existence autonome qui serait radicalement coupée du monde extrascolaire. Les genres didactiques entretiennent ainsi des relations plus ou moins étroites et de multiples sortes avec des genres inscrits dans d'autres univers : « scientifiques », privés, professionnels... Cela impose donc, toujours dans notre perspective, de les caractériser selon leur plus ou moins grande dépendance/indépendance des sphères extrascolaires, selon leurs éventuels référents extrascolaires, selon les transformations qu'ils ont subies afin d'exister didactiquement, selon leurs effets sur les genres extrascolaires...

Il convient de noter ici que cette dimension de la référence et du mode de constitution, plus ou moins autonome, des genres renvoie à des débats importants au sein des didactiques qui portent sur l'élaboration des savoirs scolaires (voir, en-

(6) Je développe et discute cette articulation dans mon article de 2011(a), consacrée à la spécificité de la perspective didactique.

(7) Voir les travaux de référence de Guy Vincent (Vincent 1980 ; Vincent, dir. 1994). 
tre autres, Chevallard 1985/1991, Chervel 1977 ou Martinand 1986). Elle permet encore d'explorer certaines tensions à l'œuvre dans les représentations des élèves et dans leurs pratiques, par exemple entre réaliser un écrit relevant au moins en partie d'un genre extrascolaire avec les normes et les fonctions qui lui sont attachées dans ce cadre (un texte autobiographique, une lettre à un ami, un compte rendu, un résumé...) et réaliser un écrit, référant sans doute à ce genre, mais reconfiguré par le cadre scolaire, à des fins d'apprentissage et/ou d'évaluation. De ce point de vue, on peut raisonnablement penser que certains genres sont susceptibles d'engendrer plus de tensions de cette sorte que d'autres, considérés en quelque sorte comme « purement» scolaires (la dictée, par exemple)... ce qui n'empêche nullement ces derniers d'engendrer des problèmes spécifiques...

\subsection{Décrire les genres dans une perspective didactique}

Parmi la multiplicité des caractéristiques « internes » des genres ${ }^{(8)}$, certaines me paraissent particulièrement intéressantes, toujours dans une perspective didactique :

— les désignations en circulation et les conflits éventuels qui les accompagnent $^{(9)}$;

- les contenus qu'ils autorisent et mettent en scène ;

- leur organisation ;

- leur architecture (longueur, disposition, modes de marquage typographique...);

- leurs modes d'inscription matérielle (support, graphie...);

- leurs caractéristiques énonciatives (avec notamment le type et le mode de présence de l'énonciateur et de l'énonciataire...);

- leurs modalités typologiques (formes des descriptions, des argumentations...) et les formes d'articulation de celles-ci ;

- les systèmes sémiotiques mis en œuvre (verbal, iconique...) et leur articulation;

- les formes syntaxiques et leur marquage (flèches...) ;

— les unités lexicales et leur marquage (lettres, chiffres...)...

Dans ce cadre, il me semble, qu'au-delà de débats légitimes sur la pertinence de chacune de ces caractéristiques ou sur l'éventuelle expansion ou réduction de cette liste ${ }^{(10)}$, l'important réside dans l'accent porté sur la caractérisation du genre comme constellation, comme entité hétérogène. Cela permet en effet de penser sa complexité et les difficultés de gestion qui peuvent lui être attachées que ce soit en production ou en réception ${ }^{(11)}$. Cela permet encore de spécifier plus finement les différences dans l'actualisation de certains genres selon les disciplines (débat, dissertation..).

(8) Il me faut ici reconnaitre ma dette envers les travaux de Dominique Maingueneau (notamment 2004).

(9) Ecriture inventée/écriture approchée...; débat /débat philosophique/débat à visée philosophique...

(10) Ainsi certains critères sont plus ou mois spécifiques à des genres particuliers : le caractère plus ou moins ouvert des exercices, par exemple.

(11) Gestion de l'hétérogénéité, voire des tensions (Reuter 1996, 2004b...) 


\subsection{Le genre au cœur du système didactique et des configurations disciplinaires}

Mais les genres « didactiques » n'existent qu'en tant qu'ils prennent place dans le système didactique et, plus précisément encore dans une configuration disciplinaire $^{(12)}$ qui détermine leur statut. De ce point de vue, d'autres traits encore me paraissent essentiels, pour caractériser le genre dans une perspective didactique :

- sa place dans le système didactique, plus ou moins centrale, plus ou moins attachée à tel domaine ou sous-domaine disciplinaire, ce qui fait que dans certains cas on peut parler de genre « emblématique » (la dictée pour l'orthographe, par exemple);

— son statut : disciplinaire (comme objet d'enseignement et d'apprentissages), paradisciplinaire (comme outil), protodisciplinaire ${ }^{(13)}$ (de pré-requis en quelque sorte) ;

- sa fonction dominante dans le fonctionnement de la classe : plutôt disciplinaire aux sens évoqués précédemment ou plutôt de l'ordre de la régulation des relations (avec cependant l'idée que les modalités de gestion de la classe et les genres qui leur sont liés — rappels à l'ordre, incitations, menaces... - varient disciplinairement ${ }^{(14)}$ );

— sa relation aux acteurs du système didactique (genre du professeur, genre des élèves, genre partagé..) ;

— son histoire (apparition récente ou non ; plus ou moins grande stabilité formelle...);

- ses relations avec les autres genres disciplinaires ;

— ses relations avec les autres genres hors de la discipline (voir le cas du « débat » ${ }^{(15)}$ ou celui de la « dissertation »);

— sa plus ou moins grande permanence (au sein du cursus et des filières);

— son caractère consensuel ou dissensuel...

\subsection{Genres et pratiques}

Complémentairement encore, je dirais, et c'est essentiel, que les genres ne se constituent qu' au travers de pratiques ${ }^{(16)}$ (ce qui explique que nous les ayons associés dans le cadre du programme de recherches mené au sein de Theodile). Cette assertion, quelque peu brutale, nécessite sans doute quelques précisions. Cela signifie que genres et pratiques se formatent mutuellement et sont interdé-

(12) Nous avons avancé la notion de configuration disciplinaire pour désigner les différentes modalités d'actualisation des disciplines selon les pays, les époques, les espaces, les moments du cursus, les filières, les pédagogies... (Reuter, Lahanier-Reuter 2004/ 2007).

(13) Je reprends ici les catégories de Chevallard (1985/1991).

(14) Ce qui demeure à étudier précisément.

(15) Sur toutes ces questions liées au débat, voir notamment Dias-Chiaruttini, 2010.

(16) Dans le sens où j'ai défini cette notion (Reuter,1996, 58 et $s q$.) c'est à dire en portant l'accent sur les dimensions d'historicité, de transformation-production, de complexité, d'actualisation matérielle, d'inscription institutionnelle, d'inscription biographique, de relations avec les autres pratiques, de structuration par des tensions et de maitrise toujours imparfaite. 
pendants, ce qui explique des désignations pouvant renvoyer à l'un et à l'autre : dictée, commentaire... Le genre, en tant que modèle implicite ou explicite, détermine les pratiques qui le génèrent; les pratiques produisent le genre en tant qu'exemplaires et en tant que catégorie ; leurs interactions assurent leur inscription dans une sphère socio-institutionnelle dont ils dépendent et qui les contraint. Dans cette perspective, la distinction - relativement labile - entre genres et pratiques a pour intérêt principal de porter l'accent, pour le premier terme, sur l'appréhension et la catégorisation d'objets, de produits... et, pour le second terme, sur l'appréhension et la catégorisation des activités, de la production, du « faire » des acteurs, facilitant ainsi — du moins je l'espère — la description et l'analyse de ces deux entités en ce qu'elles sont relativement autonomisables et en ce qu'elles sont en constante interaction dans les espaces sociaux. L'articulation entre genres et pratiques peut ainsi permettre de préciser le mode de mise en œuvre des genres (qui les sollicite ? qui les produit? s'agit-il de les écrire, de les lire, de les commenter?...), leur mode de fonctionnement, leur fonctionnalité...

C'est donc, à mon sens, au travers des relations avec les pratiques et de la fonctionnalité qui leur est associée que les genres se constituent en tant qu'éléments structurels des principaux fonctionnements didactiques, facilitant ou entravant à des degrés divers l'instauration et le maintien des conditions d'étude, la mise en circulation de contenus, la mise en œuvre de l'enseignement et des apprentissages, la réalisation des procédures évaluatives. En d'autres termes, ils contribuent à la régulation des relations entre acteurs, à la régulation des relations entre acteurs et contenus, à l'organisation du travail des sujets didactiques en lui donnant forme (les genres font faire de telle manière...), à la spécification de l'enseignement, des apprentissages et de l'évaluation...En d'autres termes encore, dans une perspective didactique, les genres ont une dimension transactionnelle, actualisée par les pratiques, entre contenus et acteurs, entre contenus, travail d'enseignement et travail d'apprentissage.

\section{De quelques usages de la notion de genre}

Afin d'étayer les intérêts que présente la notion de genre en didactique(s), je présenterai, toujours aussi brièvement, quelques exemples de recherches, menées au sein de Theodile, pour lesquelles elle a constitué et constitue encore un outil particulièrement opératoire. J'ai regroupé ces exemples, pour des raisons d'exposition, autour de trois axes : 1'exploration des configurations disciplinaires, les modes de construction des genres didactiques et l'analyse des fonctionnements et des dysfonctionnements des élèves.

\subsection{L'exploration des configurations disciplinaires}

Le premier axe rassemble les recherches que nous avons menées et que nous menons afin de spécifier les configurations disciplinaires, de mieux comprendre comment fonctionnent l'enseignement et les apprentissages au sein d'une discipline donnée.

Ainsi le système des genres et des pratiques, en ce qu'il est propre à chaque 
discipline, permet d'approcher la "matrice disciplinaire » et ses changements ${ }^{(17)}$. Ce système détermine aussi les modes d'existence des contenus, qui n'existent qu'inscrits dans des genres et des pratiques, par exemple, la lecture comme réponse à des questions (Guernier 1998) ou le commentaire valorisant à partir d'extraits. Il formate encore le travail des enseignants ${ }^{(18)}$ et celui des élèves (temps consacré, répartition du travail entre la classe et le domicile, modes d'effectuation, postures sollicitées...). Il structure aussi les sujets didactiques et leurs relations : par exemple, les «bons » élèves en français ont tendance à accepter plus facilement que les autres que l'enseignant leur demande de modifier leurs écrits, les constituant ainsi plus en genres d'apprentissage qu'en genres d'expression. La caractérisation la plus précise possible de chacun des genres et des pratiques mis en place est donc essentielle, qu'il s'agisse de la rédaction (Reuter 1996), des analyses de cas, du dossier statistique ou du paragraphe argumenté ${ }^{(19)} \ldots$

\subsection{Les modes de constitution des genres et des pratiques}

Le second axe regroupe les recherches sur les sources et les mécanismes de constitution des genres et des pratiques : cela a porté, par exemple, sur les commentaires, les « textes fondateurs » (Denizot, 2008 et 2010), l'écriture d'invention (Daunay et Denizot 2004)... mais il serait tout aussi intéressant d'explorer, par exemple, l'émergence du brouillon scolaire lié à l'accent porté sur la réécriture...

\subsection{Fonctionnements et dysfonctionnements}

Le troisième axe, celui de l'analyse des fonctionnements et des dysfonctionnements (Reuter, 2005) des élèves, auquel j'accorde beaucoup d'importance, regroupe des recherches qui tentent de mieux comprendre en quoi les genres (et les pratiques) sollicités peuvent faciliter les activités des élèves ou, au contraire, engendrer des problèmes qui leur sont, en quelque sorte, structurellement attachés. Sur le premier plan, on peut ainsi renvoyer aux travaux de Marie-Christine David-Chevalier (2006), qui ont pu établir que l'écriture de descriptions en CM2 est facilitée (et améliorée) par des consignes induisant un cadrage générique à la différence de celles n'y renvoyant pas. Sur le second plan, on peut mentionner les travaux que j'ai effectués autour de la rédaction (1996) en montrant que la «sommairisation » des écrits est, en quelque sorte, imposée par le genre et les pratiques associées (avec notamment ses contraintes temporelles) ou sur l'écriture de recherche en formation (2004b) en analysant les problèmes soulevés par la double position tendanciellement contradictoire sollicitée : chercheur et apprenti-chercheur. On peut aussi renvoyer aux recherches de Marie-France

(17) Voir, par exemple en français, la rupture de la matrice constituée par le discours magistral de l'enseignant associé aux exercices de grammaire (type Bled) et aux commentaires d'extraits (type Lagarde et Michard).

(18) Voir, par exemple, les recherches menées au sein de Theodile, sur les écrits professionnels des enseignants (Daunay, dir. 2011).

(19) Ces genres ont fait l'objet de communications, au sein du séminaire Theodile, d'Anne-Marie Jovenet, de Dominique Lahanier-Reuter et de Gérard Bécousse... 
Bishop $(2004,2011)$ qui met au jour la complexité des contrats de communication de l'écriture de soi scolaire. On peut encore renvoyer aux travaux menés, dans le cadre d'une recherche financée par l'ANR, sur les littéracies universitaires et les problèmes qu'elles soulèvent (Daunay, Lahanier-Reuter, 2011 ; Delcambre, Lahanier-Reuter, 2010)... Ainsi, dans cette perspective, la notion de genre permet d'ouvrir la palette des possibles explicatifs quant aux dysfonctionnements en éclairant, entre autres, les difficultés liées à la nouveauté des genres proposés, à l'entrée dans une culture disciplinaire, à la complexité et à des tensions structurelles ou encore à des transferts inappropriés...

\section{De quelques problèmes méthodologiques et de quelques pistes}

Il me semblerait regrettable de conclure une contribution liée à des recherches sans soulever certains des problèmes méthodologiques auxquelles elles peuvent être confrontées. Je me contenterai d'en présenter trois, avant d'insister sur trois pistes de travail qui me paraissent, aujourd'hui, particulièrement fructueuses.

\subsection{Problèmes}

Je n'évoquerai donc ici que trois des problèmes cruciaux que nous rencontrons. Le premier est celui de la constitution du corpus qui est pris dans une tension structurelle, constamment mentionnée par ceux qui ont travaillé sur cette question, entre la volonté de ne pas pré-définir le genre et la difficulté à échapper à toute pré-définition (ne serait-ce que pour constituer le corpus).

Le deuxième problème est celui de l'échelle la plus adaptée à la saisie du genre, entre macro-catégories (Marie-France Bishop parle, par exemple, à propos des écritures de soi à l'école, d'hyper-genre) et micro-catégories (par exemple, le souvenir d'une soirée vécue), ce qui impose, complémentairement, de penser les choix et/ou les changements d'échelle, le plus souvent nécessités par les recherches, leurs justifications et leurs modalités d'articulation.

Le troisième problème est celui de la catégorisation et de l'analyse des pratiques associées aux genres, ce qui renvoie aux débats sur l'analyse des activités, des actions, du travail ${ }^{(20)} \ldots$

\subsection{Trois pistes de travail}

Trois pistes de travail m'apparaissent aujourd'hui de plus en plus intéressantes aussi bien d'un point théorique que d'un point de vue méthodologique.

La première est l'analyse articulée de ce qu'un genre prescrit et proscrit, rend possible et impossible (le second aspect étant trop souvent négligé, à mon sens). Elle me parait indispensable pour cerner ses contours, préciser ses caractéristiques et éclairer d'éventuels problèmes qu'il est susceptible d'engendrer.

La deuxième piste s'incarne pour moi dans une attention privilégiée aux gen-

(20) Voir, sur cette question, le dossier des Cahiers Theodile, $\mathrm{n}^{\circ} 6$ (décembre 2005). 
res qui apparaissent et à ceux qui disparaissent ainsi qu'aux débats qui les accompagnent en ce que ceux-ci sont particulièrement révélateurs du système des positions qui justifient une configuration générique ${ }^{(21)}$.

La troisième et dernière piste que j'évoquerai pour conclure cet article est celle de la délimitation et de la formalisation de l'unité genre-pratique(s). Je l'avais proposée pour la première fois explicitement dans ma synthèse de l'ouvrage consacré aux écrits professionnels des enseignants (Reuter, 2011b, p.189) : il s'agit de considérer que tout genre didactique se constitue comme une entité reliant des contenus et des modes d'organisation à des interactions entre enseignants et élèves. Dans cette perspective, ce qu'on appelle souvent genre serait peut-être à appréhender comme un sous-genre. Ainsi, pour reprendre l'exemple que j'avais examiné, on pourrait considérer que consigne, production de l'élève et corrections de l'enseignant sont trois composantes d'un unique genre scolaire qui va s'actualiser sous des formes différentes selon les pédagogies et les disciplines.

Mais cela reste à débattre... comme tout le reste d'ailleurs.

\section{Bibliographie}

ADAM, J.-M. (1999) : Linguistique textuelle. Des genres de discours aux textes, Paris, Nathan.

BERNIÉ, J.-P. (2001) : « Genres discursifs scolaires, genres de l'activité et conceptualisation », dans Bernié J.-P., éd. : Apprentissages, développement et signification, Pessac, Presses Universitaires de Bordeaux, 155-171.

BISHOP, M.-F. (2004) : Les écritures de soi à l'école de 1850 à 2004. Places, fonctions et enjeux, Thèse de Doctorat, Université Charles-de-Gaulle - Lille III. - (2011) : "Racontez vos vacances... », Histoire des écritures de soi à l'école primaire (1882-2002), Grenoble, Presses Universitaires de Grenoble.

Bronckart, J.-P., BotA, C. (2011) : Bakhtine démasqué. Histoire d'un menteur, d'une escroquerie et d'un délire collectif, Genève, Droz.

CHERVEL, A. (1977) : ... et il fallut apprendre à écrire à tous les petits français. Histoire de la grammaire scolaire, Paris, Payot.

Chevallard, Y. (1985/1991) : La transposition didactique. Du savoir savant au savoir enseigné, Grenoble, La Pensée sauvage.

Cohen-Azria C., Sayak N., dir. (2009) : Questionner l'implicite. Les méthodes de recherche en didactiques (3), Villeneuve d'Ascq, Presses Universitaires du Septentrion.

(21) Voir, ici encore, les travaux de Nathalie Denizot. 
Daunay, B., Denizot, N. (2004) : «Construction d'un “objet didactique” ; 1'écriture d'invention au collège et au lycée. Compte-rendu d'une recherche en cours », Les Cahiers THEODILE, n 4, Université Charles-de-Gaulle - Lille III, 81-101.

DAUnAY, B., LAHANIER-REUTER, D. (2011) : « Les genres d'écrits dans la formation supérieure : étude comparative en formation professionnelle des enseignants et en formation universitaire générale », Bulletin suisse de linguistique appliquée (VALS-ASLA) , $\mathrm{n}^{\circ} 93$, Les compétences langagières dans la formation et dans le vie professionnelle - Transitions et transformations, Neuchâtel, Université de Neuchâtel, 101-113.

DAUNAY, B., dir. (2011) : Les écrits professionnels des enseignants. Approche didactique, Rennes, Presses Universitaires de Rennes.

DAVID-CHEVALIER, M.-C. (2006) : « Le référent "genre" dans l'écriture descriptive : outil ou obstacle pour l'élève ? ", Les Cahiers THEODILE, n 6 , Université Charles-de-Gaulle - Lille III, 77-90.

Delcambre, I. (2007/2010 a) : " Genres du discours », dans Reuter Y., dir. : Dictionnaire des concepts fondamentaux des didactiques, Bruxelles, De Boeck, 117-122.

— $(2007 / 2010$ b) : «Pratiques langagières », dans Reuter Y., dir. : Dictionnaire des concepts fondamentaux des didactiques, Bruxelles, De Boeck, 167172.

DelCAMBRE, I., LAHANIER-REUTER, D. (2010) : « Les littéracies universitaires : influence des disciplines et du niveau d'étude dans les pratiques de l'écrit », dans Blaser, C., Pollet, M.-C., dir. L'appropriation des discours universitaires, Namur, Diptyque, ${ }^{\circ} 18$, Presses Universitaires de Namur, 11-42.

DELCAMBRE, I., REUTER, Y. (2002) : «Images du scripteur et rapports à l'écriture », Pratiques, $\mathrm{n}^{\circ} 113-114$, Images du scripteur et rapports à l'écriture, Metz, CRESEF, 7-28.

DENIZOT, N. (2008) : Genres littéraires et genres textuels en classe de français : scolarisation, construction, fonctions et usages des genres dans la discipline français, Thèse de Doctorat, Université de Lille 3.

- (2010) : «Genres littéraires et genres textuels dans la discipline français », Pratiques, $\mathrm{n}^{\circ}$ 145-146, Didactique du français-1, Metz, CRESEF, 211-230.

DiAs-CHIARUTTINI, A. (2010) : Le débat interprétatif dans l'enseignement de la lecture et de la littérature à l'école, Thèse de doctorat, Université de Lille 3.

Dolz, J., Schneuwly, B. (1998) : Pour un enseignement de l'oral. Initiation aux genres formels à l'école, Paris, E.S.F.

ENJEUX (2001), $\mathrm{n}^{\circ}$ 50, Rapports à l'écriture, Namur, CEDOCEF, mars.

GUERNIER, M.-C. (1998) : Discours sur l'école. Etude longitudinale et comparative de discours d'élèves et de maîtres de cycle III du primaire et de sixième du collège, Thèse de Doctorat, Université Stendhal - Grenoble III.

JAUBERT, M. (2007) : Langage et construction de connaissances à l'école en sciences, Pessac, Presses universitaires de Bordeaux.

JAUBERT, M., REBIÈRE, M., BERNIÉ, J.-P. (2003) : «L'hypothèse “communauté discursive" : d'où vient-elle ? où va-t-elle ? », Les Cahiers THEODILE, n 4 , Université de Lille 3, 51-80.

LAHANIER-REUTER, D. (2005) : «Genres dans une classe de mathématiques. Première approche », Les Cahiers THEODILE, n 5, Université de Lille 3, 33-48. 
LAHANIER-REUTER, D., RODITI, E., dir. (2007) : Questions de temporalité. Les méthodes de recherche en didactiques (2), Villeneuve d'Ascq, Presses Universitaires du Septentrion.

LES CAHIERS THEODILE (2006), $\mathrm{n}^{\circ}$ 6, université de Lille 3.

Maingueneau, D. (2004) : Le discours littéraire. Paratopie et scènes d'énonciation, Paris, Armand Colin.

MARTINAND, J.-L. (1986) : Connaître et transformer la matière, Berne, Peter Lang.

PERRIN-GLORIAN, M.-J., REUTER, Y., dir. (2006) : Les méthodes de recherche en didactiques, Villeneuve d'Ascq, Presses Universitaires du Septentrion.

PRATIQUES (1995) : n 86, Lecture/écriture, Metz, CRESEF, juin.

- (1998) : $\mathrm{n}^{\circ}$ 99, La description, Metz, CRESEF, septembre.

- (2002) : $\mathrm{n}^{\circ}$ 113-114, Images du scripteur, Metz, CRESEF, juin.

REUTER, Y. (1996) : Enseigner et apprendre à écrire. Construire une didactique de l'écriture, Paris, E.S.F.

- (2004a) : "Analyser la discipline », La Lettre de la DFLM, $n^{\circ} 35$, Le français : discipline singulière, plurielle ou transversale?, Namur, 5-12.

- (2004b) : "Analyser les problèmes de 1'écriture de recherche en formation ", Pratiques, $\mathrm{n}^{\circ} 121 / 122$, Les écrits universitaires, Metz, CRESEF, juin, 9-27.

- (2005) : «Définition, statut et valeurs des dysfonctionnements en didactique », Repères, $\mathrm{n}^{\circ} 31$, L'évaluation en didactique du français, Paris, INRP, 211-231.

- (2007) : «Statut et usages de la notion de genre en didactique(s) », Le Français aujourd'hui, ${ }^{\circ} 159$, Les genres : corpus, usages, pratiques, Paris, Armand Colin, 11-18.

- (2011a) : «Penser la perspective didactique : la question de l'articulation entre disciplinaire, pédagogique et scolaire ", dans Daunay, B., Reuter, Y., Schneuwly, B., dir., Les concepts et les méthodes en didactique du français, Namur, Presses Universitaires de Namur, 35-60.

- (2011 b) : «Les écrits professionnels des enseignants : questions pour les didactiques », dans Daunay B., Les écrits professionnels des enseignants, Approche didactique, Rennes, Presses Universitaires de Rennes, 185-190.

— éd. (1994) : Les interactions lecture-écriture, Berne, Peter Lang.

— éd. (1998) : La description. Théories, recherches, formation, enseignement, Villeneuve d'Ascq, Presses universitaires du Septentrion.

— dir. (2007) : Dictionnaire des concepts fondamentaux des didactiques, Bruxelles, De Boeck.

REUTER, Y. , LAHANIER-REUTER, D. (2004/2007) : «L'analyse de la discipline : quelques problèmes pour la recherche en didactique », dans Falardeau, E., Fisher, C., Simard, C., Sorin, N., dir. : La didactique du français. Les voies actuelles de la recherche, Québec, Presses de l'université Laval, 27- 42.

SCHNEUWLY, B. (1994) : «Genres et types de discours : considérations psychologiques et ontogénétiques ", dans Reuter Y., éd. : Les interactions lecture-écriture, Berne, Peter Lang, 155-173.

VINCENT G. (1980) : L'école primaire française, Lyon, Presses universitaires de Lyon.

—éd. (1994) : L'éducation prisonnière de la forme scolaire? Scolarisation et socialisation dans les sociétés industrielles, Lyon, Presses universitaires de Lyon. 